\title{
Highly ordered CdS nanoparticle arrays on silicon substrates and photoluminescence properties
}

\author{
Y. Lei $^{\mathrm{a})}$ \\ Forschungszentrum Karlsruhe, Institut für Nanotechnologie, Karlsruhe, Germany 76021 \\ and Singapore-MIT Alliance, 4 Engineering Drive 3, Singapore 117576, Singapore \\ W. K. Chim \\ Singapore-MIT Alliance and Department of Electrical \& Computer Engineering, \\ National University of Singapore, 4 Engineering Drive 3, Singapore 117576, Singapore \\ H. P. Sun \\ Department of Materials Science and Engineering, The University of Michigan, \\ Ann Arbor, Michigan 48109 \\ G. Wilde \\ Forschungszentrum Karlsruhe, Institut für Nanotechnologie, Karlsruhe, Germany 76021
}

(Received 29 November 2004; accepted 4 January 2005; published online 1 March 2005)

\begin{abstract}
Highly ordered cadmium sulphide (CdS) nanoparticle (NP) arrays were fabricated on silicon ( $\mathrm{Si}$ ) substrates using ultrathin alumina membranes as evaporation masks. The CdS NPs are polycrystalline and are composed of ultrasmall closely packed nanocrystallites. These crystallites increase in size as the duration of the $\mathrm{CdS}$ evaporation process increases. When the thickness of the NPs changes from about 10 to $50 \mathrm{~nm}$, the size of the crystallites increases from about 5-14 to 20-40 nm. Photoluminescence measurements on the CdS NP arrays show a strong emission spectrum with two subbands that are attributed to band-edge and surface-defect emissions. The peak position and width of the band-edge emission band are closely related to the size of the crystallites in the CdS NPs. (C) 2005 American Institute of Physics. [DOI: 10.1063/1.1869545]
\end{abstract}

Nanometer-sized semiconductor particles, the so-called nanoparticles (NPs), have attracted much interest for the last two decades as they possess unique physical and optical properties that are closely related to their size. ${ }^{1-13}$ Semiconductor NPs are expected to exhibit quantum confinement effects when their size becomes comparable to the Bohr exciton radius, which results in an increase in the energy gap relative to that of the bulk solid., ${ }^{2,3}$ Among a variety of nanosized semiconductors, cadmium sulphide (CdS) has been intensively studied ${ }^{3-13}$ due to advantages such as a band-gap energy in the visible region $\left(E_{g}=2.5 \mathrm{eV}\right)$ and a relatively simple fabrication process. CdS NPs often present novel properties and have been widely used in solar cells, optoelectronics, and microelectronics. For the device application of CdS NPs, such as in electronic and optical areas, it is usually desirable to fabricate a large area array of CdS NPs. In many cases, high regularity of the CdS NP arrays is required to obtain precise control on the resultant device properties. Recently, structurally ordered CdS NPs have been fabricated in the pores of mesoporous materials. ${ }^{5,6}$

As a well-established nanotemplate, porous alumina membranes have been widely used in the fabrication of many kinds of ordered nanostructures, ${ }^{14-20}$ especially after a great improvement in their pore regularity achieved using a twostep anodization process as proposed by Masuda. ${ }^{15}$ The porous alumina membrane can be attached to a suitable substrate to act as a nanotemplate for fabricating ordered nanostructures. Using ultrathin alumina membranes (UTAMs) as etching masks, nanohole arrays have been produced on various semiconductor materials, including

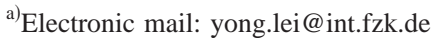

$\mathrm{GaAs}^{21}{ }^{21} \mathrm{InP},{ }^{22} \mathrm{Si}^{23}$ and $\mathrm{ZnTe}^{24}$ Recently, using UTAMs as evaporation masks, we demonstrated the fabrication of ordered carbon nanotube arrays, initiated from ordered metal catalyst arrays, on Si substrates. ${ }^{25}$ In this letter, we report the realization of highly ordered large-scale CdS NP arrays on $\mathrm{Si}$ substrates using UTAMs as evaporation masks. The detailed structure and the photoluminescence (PL) properties of these arrayed CdS NPs are also discussed. It will be shown that by changing the evaporation duration (the thickness of the deposited NPs), and hence varying the size of the crystallites in the NPs, it is possible to change the PL properties of the CdS NPs, including the peak position and width of the PL bands.

The fabrication details of UTAMs on Si substrates can be found in our previous publication. ${ }^{25}$ After mounting of the UTAM on the substrate, thermal evaporation of CdS was carried out using an evaporation rate of approximately $0.2-0.3 \mathrm{~nm} / \mathrm{s}$. After completion of the evaporation, the UTAM was removed from the substrate using acetone, leaving the highly ordered CdS NP array on the surface of the substrate. Figure 1 shows the scanning electron microscope image of a typical NP array on a Si wafer, where part of the UTAM is left behind intentionally. The regularity of the pore arrays of the UTAM and the consequent CdS NPs is very high. The typical area of the UTAM is about $1.5 \mathrm{~cm}$ $\times 1.5 \mathrm{~cm}$ with our current setup and can be as large as $20-40 \mathrm{~cm}^{2}$ with modified setup. The diameter of the deposited NPs has a monodisperse size distribution and can be adjusted from about 10 to $200 \mathrm{~nm}$. In addition, there is a sieve-like CdS film formed on top of the UTAM.

A set of two samples (samples A and B) were characterized using $\mathrm{X}$-ray diffraction (XRD), high resolution transmission electron microscopy (HRTEM), and PL measurements. The two samples were fabricated with identical UTAMs and 


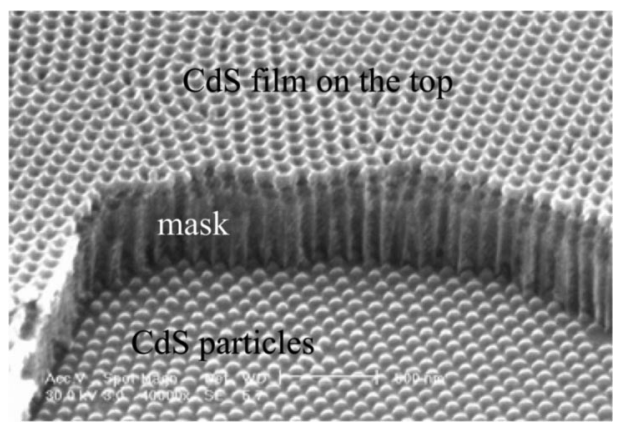

FIG. 1. CdS nanoparticle arrays on Si substrates with part of the UTAM remaining. The average diameter and spacing of the nanoparticles are about 80 and $105 \mathrm{~nm}$, respectively.

almost similar CdS evaporation conditions, except for the duration of the evaporation process which changes the thickness of the deposited films, i.e., the thickness of the NPs. The film thickness of samples A and B are about 10 and $50 \mathrm{~nm}$, respectively. The substrates for these samples are $n$-type (001) $\mathrm{Si}$ covered with a 3-nm-thick silicon dioxide $\left(\mathrm{SiO}_{2}\right)$ layer.

Figure 2 shows the microstructure of the CdS NPs of sample A. Figure 2(c) is the cross-sectional TEM image of four arrayed $\mathrm{CdS}$ NPs on the $\mathrm{SiO}_{2} / \mathrm{Si}$ substrate before removal of the UTAM. It can be seen that the NPs are located at the bottom of the apertures of the UTAMs and on the surface of the substrate. The thickness of the NPs is about 10 $\mathrm{nm}$. Most NPs are flat disc-like particles. XRD measurement [Fig. 2(d)] indicates that the evaporated NPs are hexagonal CdS [indexed as Wurtzite CdS, S. G.: P6 ${ }_{3}$ mc (186)]. Moreover, the CdS NPs exhibit a (002) preferred orientation, as revealed by the intensity ratio distribution among the diffraction peaks. It has been reported that thermally evaporated CdS films show a (002) preferred orientation as a natural result of the CdS thermal evaporation process. ${ }^{7,8}$ The width of the XRD peaks, especially the strongest peak of (002) at $26.5^{\circ}$, suggests a relatively small size of the $\mathrm{CdS}$ crystallites. The crystallite size of about $10 \mathrm{~nm}$ can be estimated from the width of the (002) peak by the Sherrer's formula. ${ }^{26}$ This size is much smaller than the planar-size of the CdS NPs of about $50 \mathrm{~nm}$, which possibly suggests that each CdS NP is a polycrystalline particle.
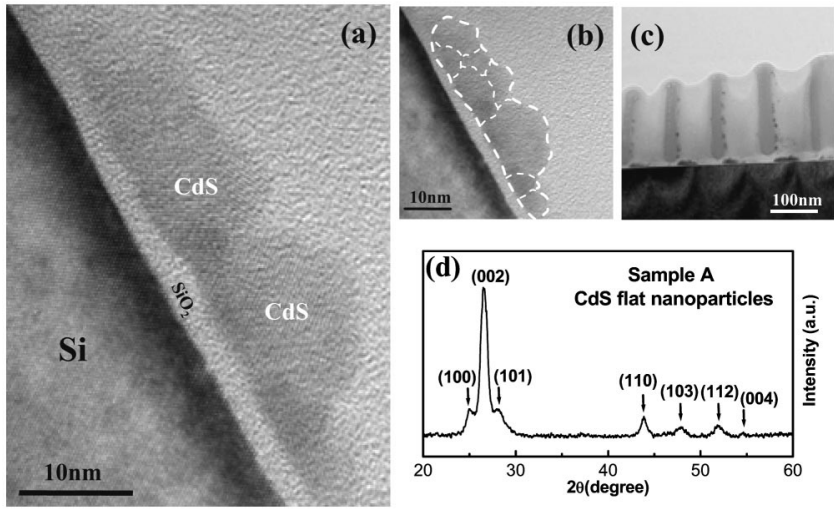

FIG. 2. Microstructures of CdS flat nanoparticles (sample A) studied by cross-sectional HRTEM and XRD measurement. (a) A CdS particle on the surface of $\mathrm{SiO}_{2} / \mathrm{Si}$. (b) Crystal-size estimation of the polycrystalline CdS particle in (a). (c) Low-magnification image of arrayed CdS nanoparticles and the UTAM. (d) XRD spectrum of sample A.
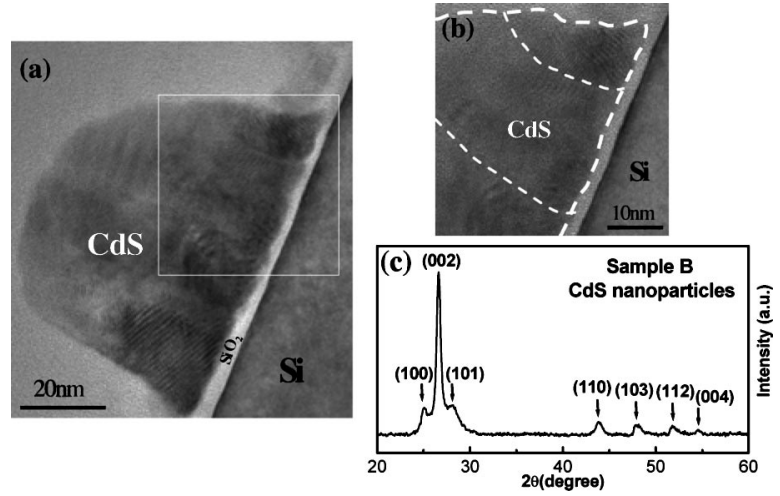

FIG. 3. Microstructures of CdS nanoparticles (sample B) studied by crosssectional HRTEM and XRD measurement. (a) A CdS particle on the surface of $\mathrm{SiO}_{2} / \mathrm{Si}$. (b) Crystal-size estimation of the polycrystalline CdS particle in the squared area of (a). (c) XRD spectrum of sample B.

Cross-sectional HRTEM measurement [Fig. 2(a)] of a $\mathrm{CdS}$ NP of sample A indicates the polycrystalline nature of the NPs. We carried out the crystal-size estimation of the NPs in Fig. 2(a) by drawing the approximate crystallite boundaries in Fig. 2(b). Eight crystallites appeared in the cross-section plane of the NP. Thus a single NP is estimated to be composed of about 30-40 closely packed small crystallites. ${ }^{27}$ The mean sizes of these crystallites are in the range of $5-14 \mathrm{~nm},{ }^{28}$ which is in good agreement with the estimated size of about $10 \mathrm{~nm}$ from the XRD measurement.

Figure 3 shows the microstructure of the CdS NPs of sample B. It was found that, as the thickness of the CdS NPs increases from about 10 to $50 \mathrm{~nm}$, the shape of the NPs changes from flat discs to hemispheres. One of the hemispherical NPs is shown in Fig. 3(a). This shape change of the evaporated NPs is caused by a closure effect of the UTAM pores during the evaporation process. ${ }^{25}$ XRD measurement [Fig. 3(c)] of sample B confirms the (002) preferred orientation of the CdS NPs. The (002) XRD peak is apparently narrower than that of sample A, which implies that the NPs of sample B are composed of relatively larger crystallites. This is confirmed by the crystal-size estimation in Fig. 3(b), which is an enlarged image of a squared area in Fig. 3(a). The mean sizes of the crystallites of the NPs in sample B are in the range of $20-40 \mathrm{~nm},{ }^{28}$ which are apparently larger than that in sample A. This suggests that, as the evaporation progresses and the thickness of the NPs increases, the crystallite size also increases.

Room temperature PL spectra of samples A and B are shown in Fig. 4. We carried out a multipeak Gaussian fit to the PL spectra and found that both spectra consisted of two Gaussian fitted peaks (denoted as subbands I and II). Subbands I and II of sample A are centered at about 473 and 575 $\mathrm{nm}$, respectively [Fig. 4(a)], while those of sample B are centered at about 506 and $563 \mathrm{~nm}$, respectively [Fig. 4(b)].

According to previous studies, ${ }^{3-13}$ generally there are two PL emissions of CdS NPs, namely band-edge and surface-defect emissions. Band-edge emission is ascribed to the radiative recombination of excitons in the NPs. The PL peak energy of such band-edge emission is usually slightly lower than the band-gap energy of the CdS NPs. It is known that the band-gap energy of the NPs is related to the quantum confinement effect of excitons in the NPs. In the "quantum confinement region," which is defined as the region with particle (crystallite) size smaller than 4 times the Bohr exci- 


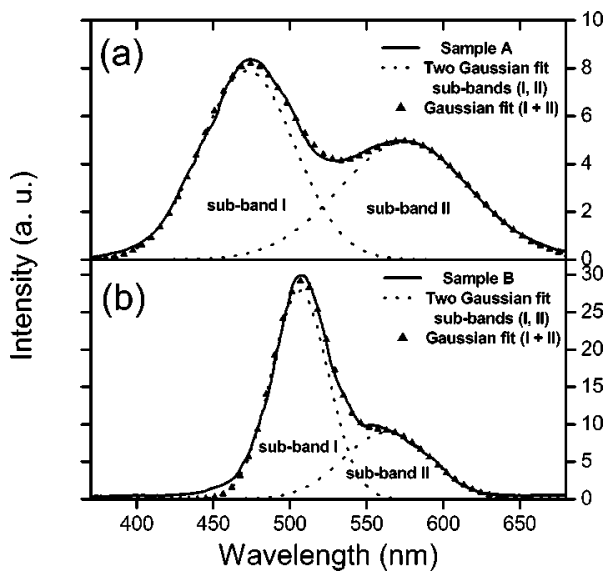

FIG. 4. PL spectra of samples A (a) and B (b) and their two Gaussian fit subbands. The excitation wavelength is $350 \mathrm{~nm}$. The peak positions of the subbands I and II are located at about 473 and $575 \mathrm{~nm}$ in (a) and 506 and $563 \mathrm{~nm}$ in (b), respectively.

ton radius (about $12 \mathrm{~nm}$ for $\mathrm{CdS}$ ), $3,4,9$ the excitons in the particles are "squeezed" and the band-gap energy increases noticeably as the size of the particles decreases. In this case, the PL peak positions of the band-edge emission of the NPs are strongly size-dependent and usually in the wavelength range of 420-500 $\mathrm{nm}$ for CdS. ${ }^{4,6,9,13}$ Some ultrasmall (about $4 \mathrm{~nm}) \mathrm{CdS}$ NPs even have a band-edge emission peaked at about $400 \mathrm{~nm} \cdot{ }^{12,13}$ When the crystallite size of the CdS NPs becomes larger, the confinement of excitons becomes weaker. There is almost no exciton confinement when the crystallite size is about $50 \mathrm{~nm}$, thus resulting in band-edge emission with size-independent PL peak positions at about $500 \mathrm{~nm}$, a wavelength that is slightly smaller than that corresponding to the band-gap energy of bulk CdS. On the other hand, the surface-defect emission is caused by surface states such as sulphur vacancies and/or sulphur dangling bonds. The PL peak positions of the surface-defect emission are usually in the wavelength range of 530-580 nm., ${ }^{6,10,12}$

In our case, it is reasonable to attribute subbands I and II to band-edge and surface-defect emissions, respectively. The $473 \mathrm{~nm}$ PL peak position of subband I in sample A suggests that the CdS NPs in this sample exhibit properties in the quantum confinement region. This is in agreement with the estimated crystallite size of about 5-14 nm. The broad subband I of sample A is caused by the wide band-gap energy distribution of the crystallites in the CdS NPs, which originates from the wide size distribution $(5-14 \mathrm{~nm})$ of these crystallites. The PL peak position of subband I changes to about $506 \mathrm{~nm}$ in sample $\mathrm{B}$, and this is caused by the large crystallite size of $20-40 \mathrm{~nm}$, which lies outside the quantum confinement region. Because the band-gap energy of crystallites in this region is independent of the crystallite size, the subband I of sample B is a relatively narrow spectrum, though the crystallites in the CdS NPs also have a wide size distribution $(20-40 \mathrm{~nm})$. The $33 \mathrm{~nm}$ redshift of the peak position of subband I from sample A to B is a consequence of the increase in size of the crystallites in the CdS NPs from 5-14 $\mathrm{nm}$ in sample A to $20-40 \mathrm{~nm}$ in sample B. As for the subband II, there is a $12 \mathrm{~nm}$ blueshift of the peak position from sample A to B. It has been reported that, in the case of CdS NPs embedded in polymers, the peak position of the surface-defect PL emission of CdS NPs is weakly related to the size of the NPs. ${ }^{10,11}$ Therefore, the blueshift in our case may also be related to the size change of the crystallites in NPs.

In summary, we have fabricated large-scale ordered CdS nanoparticle arrays on Si substrates using UTAMs as evaporation masks. The fabricated CdS NPs are polycrystalline and composed of small crystallites. The size of the crystallites in the CdS NPs increases as the duration of the evaporation process increases, resulting in the red-shift of the peak position of the band-edge PL emission of the CdS NPs. It is worth noting that, by adjusting the evaporation duration (thickness of the NPs), and hence varying the size of the crystallites in the NPs, it is possible to tune the PL properties of the CdS NPs, including the peak position and width of the PL bands.

Y.L. is grateful for research fellowships from the Alexander von Humboldt Foundation and the Singapore-MIT Alliance.

${ }^{1}$ L. Banyai and S. W. Koch, Semiconductor Quantum Dots (World Scientific, Singapore, 1993).

${ }^{2}$ A. P. Alivisatos, Science 271, 933 (1996).

${ }^{3}$ Y. Kayanuma, Phys. Rev. B 38, 9797 (1988).

${ }^{4}$ L. Spanhel, M. Haase, H. Weller, and A. Henglein, J. Am. Chem. Soc. 109, 5649 (1987).

${ }^{5}$ T. Hirai, H. Okubo, and I. Komasawa, J. Phys. Chem. B 103, 129 (1999).

${ }^{6}$ W. S. Chae, J. H. Ko, I. W. Hwang, and Y. R. Kim, Chem. Phys. Lett. 365, 49 (2002).

${ }^{7}$ R. Sagar and M. P. Srivastava, Phys. Lett. A 183, 209 (1993).

${ }^{8}$ K. L. Narayanan, K. P. Vijayakumar, K. G. M. Nair, and R. Kesavamoorthy, Phys. Status Solidi A 164, 725 (1997).

${ }^{9}$ T. Orii, S. Kaito, K. Matsuishi, S. Onari, and T. Arai, J. Phys.: Condens. Matter 14, 9743 (2002).

${ }^{10}$ B. Bhattacharjee, D. Ganguli, and S. Chaudhuri, J. Nanopart. Res. 4, 225 (2002).

${ }^{11}$ L. Qi, H. Colfen, and M. Antonietti, Nano Lett. 1, 61 (2001).

${ }^{12}$ J. Zhang, L. Sun, C. Liao, and C. Yan, Solid State Commun. 124, 45 (2002).

${ }^{13}$ T. R. Ravindran, A. K. Arora, B. Balamuruganm, and B. R. Mehta, Nanostruct. Mater. 11, 603 (1999).

${ }^{14}$ C. R. Martin, Science 266, 1961 (1994).

${ }^{15}$ H. Masuda and K. Fukuda, Science 268, 1466 (1995).

${ }^{16} \mathrm{~J}$. Li, C. Paradopoulos, J. M. Xu, and M. Moskovits, Appl. Phys. Lett. 75, 367 (1999).

${ }^{17}$ K. Nielsch, F. Muller, A. P. Li, and U. Gösele, Adv. Mater. (Weinheim, Ger.) 12, 582 (2000).

${ }^{18} \mathrm{H}$. Masuda, K. Yasui, and K. Nishio, Adv. Mater. (Weinheim, Ger.) 12, 1031 (2000).

${ }^{19}$ Y. Lei, L. D. Zhang, G. W. Meng, G. H. Li, X. Y. Zhang, C. H. Liang, W. Chen, and S. X. Wang, Appl. Phys. Lett. 78, 1125 (2001).

${ }^{20}$ X. Mei, D. Kim, H. E. Ruda, and Q. X. Guo, Appl. Phys. Lett. 81, 361 (2002).

${ }^{21}$ G. Cheng and M. Moskovits, Adv. Mater. (Weinheim, Ger.) 14, 1567 (2002).

${ }^{22}$ M. Nakao, S. Oku, T. Tamamura, K. Yasui, and H. Masuda, Jpn. J. Appl. Phys., Part 1 38, 1052 (1998).

${ }^{23}$ J. Liang, H. Chik, A. Yin, and J. Xu, J. Appl. Phys. 91, 2544 (2002).

${ }^{24}$ Q. X. Guo, T. Tanaka, M. Nishio, H. Ogawa, X. Mei, and H. Ruda, Jpn. J. Appl. Phys., Part 2 41, L118 (2002).

${ }^{25}$ Y. Lei, K. S. Yeong, J. T. L. Thong, and W. K. Chim, Chem. Mater. 16, 2757 (2004).

${ }^{26}$ B. D. Cullity, Elements of X-ray Diffraction (Addison-Wesley, New York, 1959).

${ }^{27}$ In the simplified case of a round flat-disc with eight crystallites at the diameter line, there are about $30-40$ crystallites in the discs.

${ }^{28}$ The mean size of a crystallite here is defined as the diameter of a sphere with the same volume of the crystallite. 